\title{
Polyubiquitination and Proteasome Signals in Tubulobulbar Complexes of Rat Late Spermatids
}

\author{
Manaka Akashi, Sadaki Yokota, Hideaki Fujita \\ Section of Functional Morphology, Faculty of Pharmaceutical Sciences, Nagasaki International University, Nagasaki, Japan \\ Email: syokota123@gmail.com
}

Received September 20, 2013; revised October 20, 2013; accepted October 27, 2013

Copyright (C) 2013 Manaka Akashi et al. This is an open access article distributed under the Creative Commons Attribution License, which permits unrestricted use, distribution, and reproduction in any medium, provided the original work is properly cited.

\begin{abstract}
To illustrate the involvement of tubulobulbar complexes (TBC) in ubiquitin-proteasome degradation of unnecessary proteins in the head cytoplasm of late spermatids, the localization of polyubiquitin and proteasome was studied by immunofluorescence and immunoelectron microscopy. Polyubiquitin localized to TBC and proteasome subunit $\alpha$ to dense materials surrounding the TBC in the cytoplasm of Sertoli cell enwrapping sickle-shaped spermatid heads. The results suggest that the TBC is a structural device for ubiquin-proteasome degradation of unnecessary proteins in the cytoplasm of spermatid head during rapid reduction of the head cytoplasm and nuclear compaction of late spermatids.
\end{abstract}

Keywords: Tubulobulbar Complex; Polyubiquitin Signals; Proteasome; Immunoelectron Microscopy

\section{Introduction}

Spermatogenic process is divided largely into three phases: 1) the proliferative phase in which spermatogonia undergo rapid successive division, 2) the meiotic phase in which recombination and segregation of chromosomes occur, 3) the differentiation phase in which spermatids transform drastically into spermatozoa which are motile cells carrying haploid genome to the egg $[1,2]$. The third phase is called spermiogenesis, which is divided into 19 steps in rat [3], and involves the formation of acrosome and flagellum [4-6], the condensation of nucleoplasm [7, $8]$ and the elimination cytoplasm $[9,10]$. Finally, spermatids transform to sperm which are released into the lumen of seminiferous tubule. This process, spermiation, is composed of a series of complicated phenomena in which Sertoli cells deeply involve [11-13]. Russell and Clermont [14] observed precisely the isolation process of sperm head from Sertoli cell and found that a dozen tubular processes project out from the ventral concavity of the head to indent the Sertoli cell. The tubular processes extend in the cytoplasm of Sertoli cell and their apex distends to form globule (bulbus) which assembles each other. They called the structure "tubulobulbar complexes" and supposed that the structures function as a scaffold to anchor the spermatid head to the Sertoli cell [14]. It was shown that the time course of the rapid cytoplasmic reduction in late spermatid head matched with that of the formation of tubulobulbar complexes (TBC) [15]. Moreover, the activity of acid phosphatase, a lysosomal marker enzyme, was detected in the TBC and in the cytoplasm of Sertoli cell surrounding the bulbi, suggesting that the lysosomal degradation system is involved in the elimination of cytoplasm from the head region of late spermatid [16].

The intracellular degradation system is largely divided into two categories; lysosome system $[17,18]$ and ubiquitin-proteasome system $[19,20]$. In the former system, the target to be degraded is segregated by membrane to form autophagosomes which fuse later with lysosomes and the target is degraded by lysosomal enzymes [18]. In the latter system, ubiquitin molecules are covalently coupled (ubiquitinated) with the target protein which is then recognized by proteasome and degraded $[19,20]$. Although the relationship between the TBC and the lysosomal system is suggested [16], given the rapid and massive degradation of proteins in late spermatid head, it is also expected that the ubiquitin-proteasome system is involved in this degradation. Here, we study whether ubiquitin and proteasome localize to the TBC using immunofluorescence (IF) and immunoelectron micros- copy (IEM) techniques. The results clearly show that these two signals are present in the TBC, suggesting the ubiquitin-proteasome system participates in the degradation of unnecessary proteins in the head cytoplasm of late 
spermatids.

\section{Materials and Methods}

\subsection{Animals and Antibodies}

Male Wistar rats (200 g BW) were used. Testes were taken out from the animals anesthetized with ether. All experiments were performed in accordance with the Guidance for Animal Experiments issued by the Nagasaki International University. Mouse monoclonal antibody (FK2) to multi-ubiquitin chain (M-Ub) was obtained from Nippon Biotest Laboratories Inc. (Tokyo, Japan). Rabbit antibody to $\alpha$ subunit of 20S proteasome $(\mathrm{P}-\alpha \mathrm{S})$ was purchased from Merck Millipore (Darmstadt, Germany). Alexa $568^{\circledR}$ or Alexa $488^{\circledR}$-conjugated goat anti-rabbit IgG or goat anti-mouse IgG were obtained from Molecular Probes (Eugene, OR, USA). DAPI was from Hoechst (Tokyo, Japan). Protein A/G/L-gold 15-nm probe were prepared as described previously [21].

\subsection{Routine Electron Microscopy}

Testis tissue blocks from rats were fixed in the fixative consisting of $4 \%$ paraformaldehyde $(\mathrm{w} / \mathrm{v}), 1 \%$ glutaraldehyde $(\mathrm{v} / \mathrm{v}), 0.02 \% \mathrm{CaCl}_{2}(\mathrm{w} / \mathrm{v})$ and $0.05 \mathrm{M}$ Hepes$\mathrm{KOH}(\mathrm{pH} 7.4)$ overnight at $4^{\circ} \mathrm{C}$. After wash in PBS, testis tissue blocks were fixed $1 \%$ reduced osmium tetroxide $(\mathrm{w} / \mathrm{v})$ for $1 \mathrm{~h}$, dehydrated and embedded in Epon. Thin sections were contrasted with lead citrate and examined with a Hitachi electron microscope.

\subsection{Immunofluorescence (IF) Staining}

Frozen sections ( $6 \mu \mathrm{m}$ thick) of testes of rats were fixed in $4 \%$ paraformaldehyde $(\mathrm{w} / \mathrm{v})$ in $0.1 \mathrm{M}$ Hepes- $\mathrm{KOH}$ buffer $(\mathrm{pH}$ 7.4) for $15 \mathrm{~min}$. After permeation treatment with $0.1 \%$ Triton X-100 (v/v) $+0.2 \%$ Saponin $(\mathrm{w} / \mathrm{v})$, sections were incubated in $2 \%$ fish gelatin $(\mathrm{w} / \mathrm{v})$ for 30 min to block non-specific adsorption of IgG, followed by overnight incubation with mouse FK2 antibody against $\mathrm{M}-\mathrm{Ub}(\times 2000)$ or rabbit anti-P- $\alpha \mathrm{S}$ antibody $(\times 500)$. After washing with PBS, reacted IgG was visualized by Alexa $568^{\circledR}$ or Alexa $488^{\circledR}$-conjugated goat anti-mouse IgG or anti-rabbit IgG. For dual staining of $\mathrm{M}-\mathrm{Ub}$ and $\mathrm{P}-\alpha \mathrm{S}$, sections were incubated in the mixture of mouse FK2 antibody and rabbit anti-P- $\alpha \mathrm{S}$ and each reacted $\operatorname{IgG}$ was visualized by Alexa $568^{\circledR}$-conjugated goat anti-mouse $\mathrm{IgG}$ and Alexa $488^{\circledR}$-conjugated goat anti-rabbit IgG. For immunofluorescence control, non-immune serum was used instead of the primary specific antibodies. Nuclei were stained by $3 \mu \mathrm{M}$ DAPI for $60 \mathrm{~min}$ at RT. The preparations were examined with a Nikon Eclipse E600 fluorescence microscope (Nikon, Tokyo, Japan). The images were merged using Adobe Photoshop 7.0 to determine whether each antigen colocalizes to the same area. The stage of seminiferous cycle was determined from the localization of elongating and elongated spermatids with individual tubules as previously described [22].

\subsection{Immunoelectron Microscopy (IEM)}

Testes of rats were cut into small blocks in the fixative consisting of $4 \%$ paraformaldehyde $(\mathrm{w} / \mathrm{v}), 0.2 \%$ glutaraldehyde (v/v), $0.02 \% \mathrm{CaCl}_{2}(\mathrm{w} / \mathrm{v})$ and $0.1 \mathrm{M}$ Hepes$\mathrm{KOH}(\mathrm{pH} 7.4)$ and testis tissue blocks were kept in the fixative for $1 \mathrm{~h}$ at $4^{\circ} \mathrm{C}$. After wash in PBS, fixed tissue blocks were dehydrated in ethanol and embedded in LR White at $-20^{\circ} \mathrm{C}$, followed by resin polymerization under UV light at $-20^{\circ} \mathrm{C}$. Thin sections were cut with a diamond knife equipped with a Reichert Ultracut R, mounted onto nickel grids, and stored in a desiccator. Sections were treated with $2 \%$ fish gelatin in PBS $(v / v)$ and incubated with mouse FK2 antibody $(\times 2000)$ or rabbit anti-M-Ub antibody $(\times 500)$ overnight at $4^{\circ} \mathrm{C}$. Nonimmune mouse or rabbit sera were used instead of the primary specific antibodies for control. Reacted IgG was visualized by Protein A/G/L 15-nm-gold probe. Sections were contrasted with $2 \%$ uranyl acetate $(\mathrm{w} / \mathrm{v})$ and lead citrate, coated with carbon and examined with a Hitachi H7650 electron microscope (Tokyo, Japan) at an acceleration voltage of $80 \mathrm{kV}$. Stage of seminiferous tubules and step of spermatids were judged in accordance with the stages of seminiferous cycle and step of spermatids were determined as described by Russell et al. [22].

\section{Results}

\subsection{Routine Electron Microscopy of TBC}

Tubulobulbar complexes (TBC) similar to those described previously $[14,15]$ were confirmed in rat testis used in this study. The sickle-shaped heads of very late spermatids were embedded in an apical process of the Sertoli cell cytoplasm which widely spread in the ventral side but not in the dorsal side of the spermatid head. From the ventral surface of the head, very narrow tubular processes of the spermatid plasma membrane invaginated into the Sertoli cell cytoplasm (Figure 1(A)). The tubules extended to deeply into the cytoplasm and the apices of the tubules distended to form globules which coexisted with clear vesicles in the Sertoli cell (Figure 1(B)). Frequently, small protrusions of spermatid plasma membrane into the Sertoli cell were observed on the surface of the heads (Figure 1(A)). Many cross-sectioned tubules were noted in the section cut through plane running from the apex to base of spermatid head (Figure 1(B)).

\subsection{IF Staining for Multi-Ubiquitin Chain}

Staining for multi-ubiquitin chain (M-Ub) was observed 


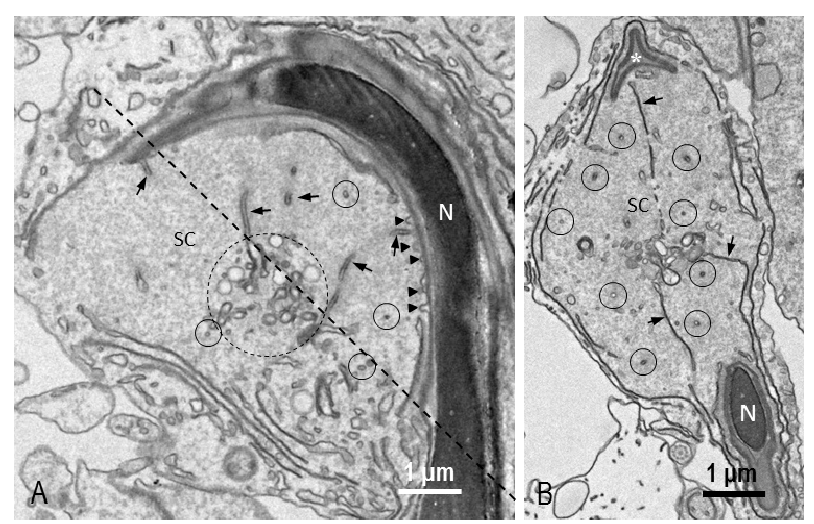

Figure 1. REM image of TBC. (A) A longitudinal section. Longitudinally-sectioned tubules (arrows) and cross-sectioned tubules (small circles) are observed in a Sertoli cell (SC). Distal ends of tubules distend and assemble to form a cluster (large circle). Small protrusions showing beginnings of the tubules are seen on the surface of sickle-shaped spermatid head (arrowheads). Oblique dotted line is a plane of section shown in B; (B) A section cut through an oblique line in A. Long tubules (arrows) extending from nucleus and from perforatorium (*) are seen. Many cross-sectioned tubules are also observed (circles). $\mathrm{N}$ : nucleus.

in the nuclei and cytoplasm of spermatogenic cells. We observed the staining during differentiation of spermatogenic cells. The nuclei and cytoplasm of spermatogonia were weakly and diffusely stained. Similar staining was noted in spermatocytes at stages I to III (Figure 2(A)). Nuclear and cytoplasmic staining increased and was highest at stage VIII (Figures 2(B)-(D)). Afterward, staining in the nuclei decreased rapidly whereas discrete granular staining increased. In spermatocytes at stage X, large spot in the nuclei was stained for M-Ub (Figure 2(E)) and at stages XII-XIII the staining intensity of this spot increased (Figure 2(F)). M-Ub-positive small granules were visible in the nuclei and cytoplasm of steps 1 8 spermatids (Figures 2(A)-(D)). In step 9 - 14 spermatids, the nucleoplasm was weakly stained but strong staining was noted in the cytoplasm (Figures 2(E) and (F)). The intensity of this cytoplasmic staining was highest at steps $12-14$, afterward decreased and eventually disappeared. Instead, moderately-sized granules in the area surrounding spermatid heads were visible, and in step 19 spermatids, these granules were located in the area contact with the ventral side of the sickle-shaped heads (Figure 2(D)). Next, we observed these granules in steps 18 and 19 spermatids at higher magnification. The granules were noticeable in step 18 spermatids and located near the tip of head (Figure 3(A)) whereas in step 19 spermatids, the granules moved caudally and were located in the central region of the ventral concavity of the sickle-shaped spermatid head (Figure 3(B)). No staining was observed in control sections.

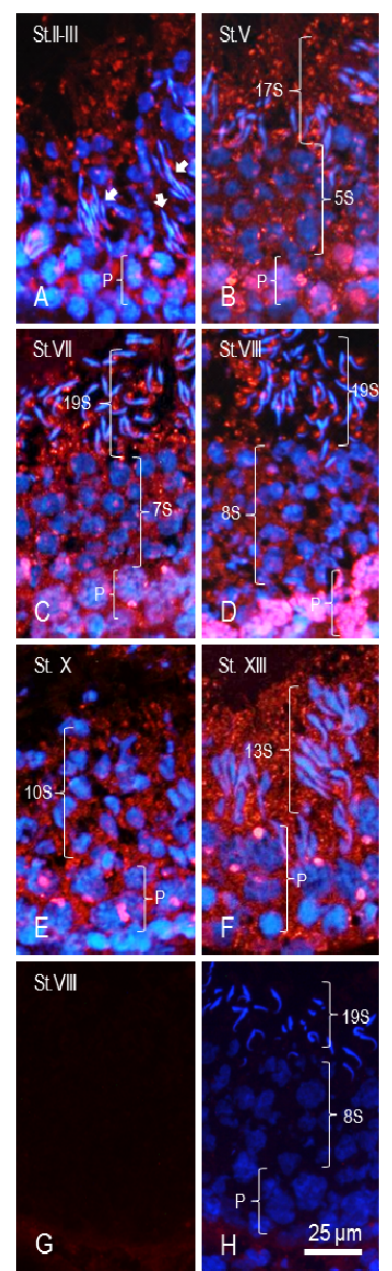

Figure 2. IF staining of M-Ub in seminiferous tubules. (A) Stage II-III. In pachytene spermatocytes (P), punctate staining in nuclei and diffuse staining in cytoplasm are observed. In elongated cytoplasm of step 16 spermatids, many granules are stained (arrows); (B) Stage V. Spotty staining in the nuclei and diffuse staining in the cytoplasm of pachytene spermatocytes (P) are observed. Dotted and diffuse staining is seen in step 5 spermatids (5S); (C) Stage VII. The nuclei and cytoplasm of pachytene spermatocytes (P) are stained. Dotted staining is seen the nuclei of step 7 spermatids (7S). Around step 19 spermatids (19S), small granules are stained; (D) Stage VIII. Pachytene spermatocytes (P) are strongly stained. In Step 8 spermatids (8S), cytoplasmic diffuse staining and granular staining are observed. Around step 19 spermatids spotty staining is seen; (E) Stage $X$. In the nuclei of pachytene spermatocytes (P), large spots are stained. The cytoplasm and nuclei of these cells and step 10 spermatids (10S) are also stained; (F) Stage XIII. In the nuclei of pachytene spermatocytes (P), large spot staining is seen. In the cytoplasm of step 13 spermatids (13S), numerous granules are stained; (G) Stage VIII. Control section without DAPI staining. Although very weak staining in connective tissue is seen, seminiferous tubule is completely negative for staining; $(\mathrm{H})$ Control section with DAPI staining. The stage is determinable. 19S: Step 19 spermatids. 13S: Step 13 spermatids. P: Pachytene spermatocytes. No M-Ub staining is noted. 

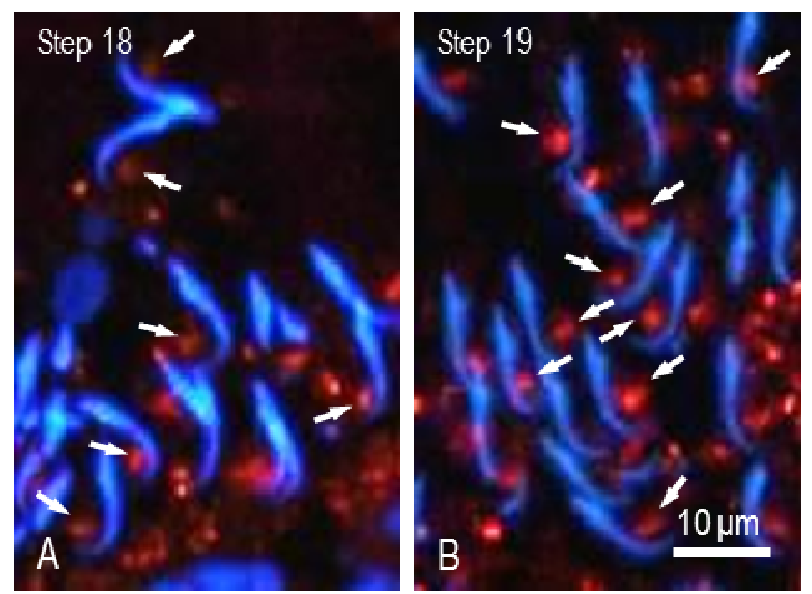

Figure 3. High power view of M-Ub staining of spermatids. (A) Sickle-shaped heads of step 18 spermatids are observed. Stained spots are located near the tip of head (arrows); (B) Heads of 19 spermatids. Stained spots moved to the center of ventral concavity of heads (arrows).

\subsection{Dual IF Staining of M-Ub and Proteasome $\alpha$-Subunit (P- $\alpha \mathbf{S})$}

Proteins coupled with M-Ub are recognized and degraded by proteasome. This means that both proteins might localize to the same place at the same time. Therefore, we observed the relationship between $\mathrm{M}-\mathrm{Ub}$ positive spots and $\mathrm{P}-\alpha \mathrm{S}$ localization in the region of the ventral concavity of the sickle-shaped spermatid heads. P- $\alpha$ S staining was observed in residual bodies of step 19 spermatids and the cytoplasm of Sertoli cells (Figure 4(B)). M-Ub spots were also stained for P- $\alpha \mathrm{S}$ (Figures (A)-(C), arrowheads). In control sections, no staining for both proteins was noted (Figure 4(D)).

\subsection{IEM Staining of M-Ub}

Since IEM localization of M-Ub in spermatogenic cells during spermatogenesis was similar to the results reported previously [23], in present study, we focused on the localization of M-Ub in the IF stained M-Ub spot at the ventral concavity of step 19 spermatid heads. Strong gold labeling showing M-Ub was associated with the lumen of tubules and dense material surrounding the tubules composing the TBC (Figures 5(A) and (B), arrowheads). Weak $\mathrm{M}-\mathrm{Ub}$ labeling was observed in the cytoplasm of Sertoli cells and the labeling in the area where clear vesicles and globules assembled together, was stronger than the other area. M-Ub was detected in subacrosomal space, perforatorium and the nucleoplasm of spermatids (Figures 5(A) and (B)). In control sections, no gold labeling was observed.

\subsection{IEM Localization of $P-\alpha S$}

Gold particles showing $\mathrm{P}-\alpha \mathrm{S}$ were observed in the cyto-
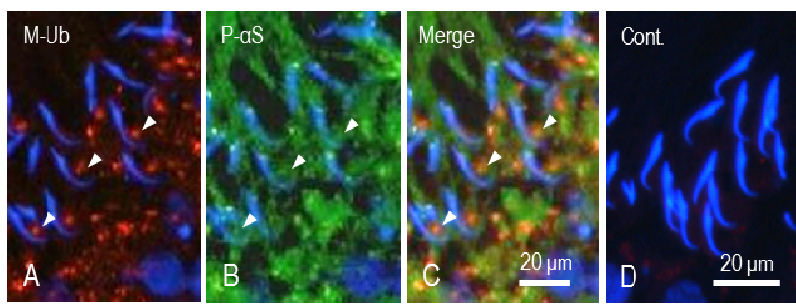

Figure 4. Dual staining of M-Ub and proteasome $\alpha$ subunit in step 19 spermatids. (A) M-Ub. Stained spots are seen in the area facing ventral concavity of sickle-shaped spermatid heads (arrows); (B) P- $\alpha$ S. Area surrounding spermatid heads are stained. Some spots are stained for both proteins (arrowheads); (C) Merged A and B. Arrowheads indicate the spots stained for both proteins; (D) Control. No staining for $\mathrm{M}-\mathrm{Ub}$ and $\mathrm{P}-\alpha \mathrm{S}$ is noted.

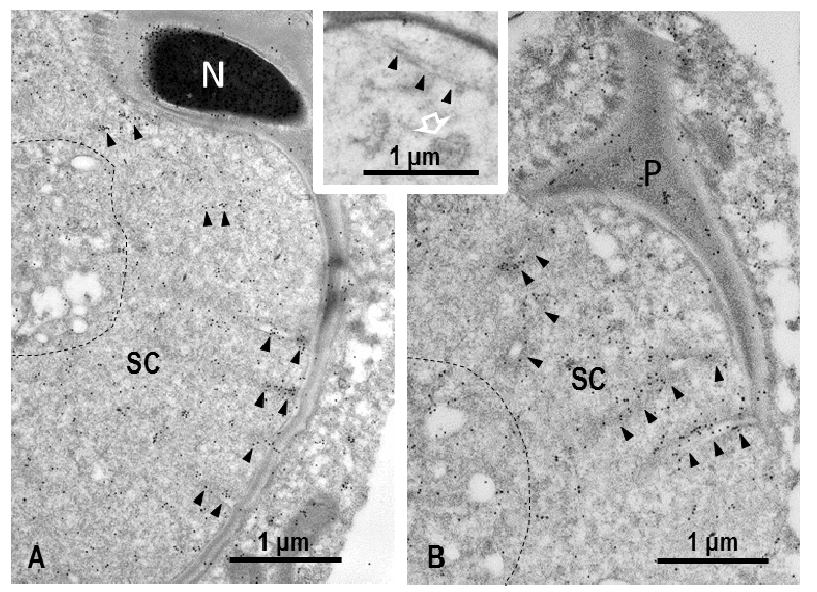

Figure 5. IEM staining of $M-U b$ in TBC in step 19 spermatids. (A) Nearly longitudinally sectioned sickle-shaped head. Gold particles showing M-Ub sites are seen on dense material around tubules and in the lumen of tubules (arrowheads). Globules of the distal end of tubules assemble together with clear vesicles (surrounded by dotted line). This area is also labeled. Gold particles are observed in the periphery of the nucleus of spermatid (N). SC: Sertoli cell; (B) Cross-sectioned tip of step 19 spermatid head. Gold labeling is observed in dense material surrounding tubules, in the lumen of tubules (arrowheads), and in cluster of tubule end and clear vesicles (surrounded by dotted line). Perforatorium (P) is also stained. SC: Sertoli cell. Inset. Control section. No gold labeling is noted in tubule (arrowheads) as well as tubule ends (white arrow).

plasm of Sertoli cells (Figure 6). If carefully observed, $\mathrm{P}-\alpha \mathrm{S}$ signals were associated with dense material around the tubules and cluster of globules composing the TBC (Figure 6). On the contrary, the lumen of clear vesicles were almost negative for P- $\alpha \mathrm{S}$ (Figure 6, *). In control sections, gold signals were noted.

\section{Discussion}

It was reported that approximately $25 \%$ of total cell body of spermatid is reduced when the spermatid is released to 


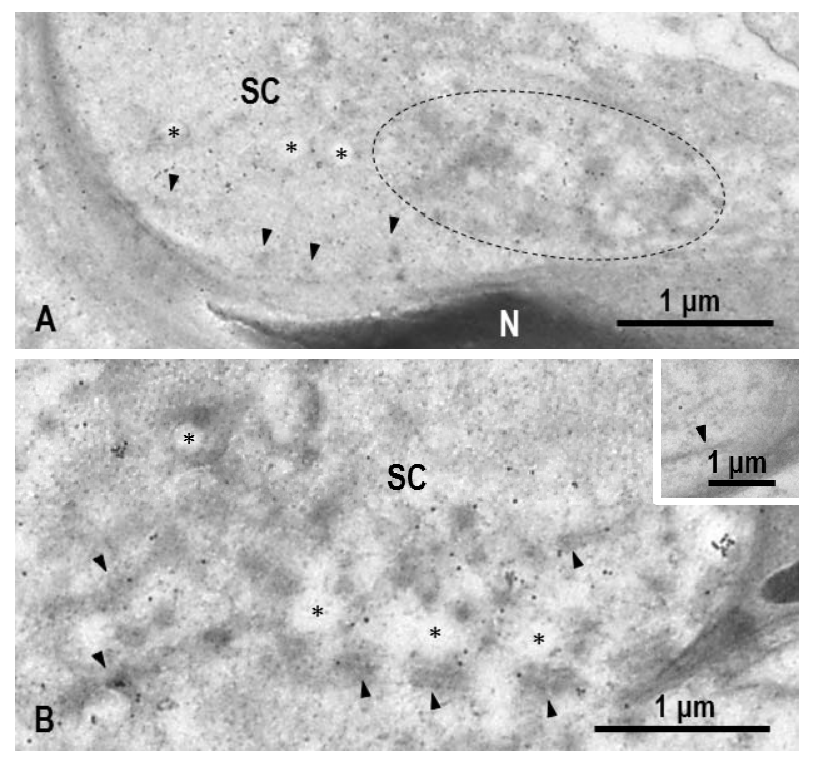

Figure 6. IEM staining of proteasome asubunit $(\mathrm{P}-\alpha \mathrm{S})$ in step 19 spermatid heads. (A) Longitudinal section of spermatid head. Gold particles showing $\mathrm{P}-\alpha \mathrm{S}$ are observed in the cytoplasm of Sertoli cell (SC). Some particles are associated with dense material surrounding tubules (arrowheads) and clear vesicles (*). Gold labeling is also present in cluster of tubule ends and clear vesicles (surrounded by dotted line). N: Nucleus of spermatid; (B) Cross-section of spermatid head. Gold labeling is seen on dense material along tubules (arrowheads) and around clear vesicles (*). SC: Sertoli cell. Inset. Control. No gold labeling is observed. Arrow indicates tubule.

the lumen of seminiferous tubule during the final stage of spermiogenesis [24]. It is completed by pinch-off of the cytoplasm of spermatids as residual bodies which are incorporated and degraded by Sertoli cells $[25,26]$. In the light of the nuclear area in step 19 spermatids, $70 \%$ of the nuclear volume is reduced as compared to that in the previous steps [16]. To accomplish such reduction, all degradation systems, including lysosomal degradation system and ubiquitin-proteasome system, are used. It is suggested that the cytoplasm casted off from spermatids is ingested by Sertoli cells and degraded in the lysosomal system [24]. Similarly, the lysosomal system of Sertoli cells is suggested to be also involved in the extensive elimination of the nuclear area through the TBC [16]. This idea is due to that the activity of acid phosphatase and a lysosomal marker enzyme is detected in the TBC [16].

In the present study, we have shown first time that multi-ubiquitin chain (M-Ub) (multi-ubiquitinated proteins) signals are associated with the TBC. The result indicates that multi-ubiquitinated proteins are present in the TBC. The multi-ubiquitinated proteins are recognized by ubiquitin receptor and trapped into proteasome to be degraded [19-20,27]. Therefore, the multi-ubiquitinated proteins in the TBC, when proteins are not clear, seem to be degraded by proteasome. In the present study we have shown that proteasome is detected in the cytoplasm of Sertoli cells and associated with dense material surrounding the TBC. The result suggests that multi-ubiquitinated proteins detected are degraded by proteasome. Since the M-Ub signals are present in the nucleoplasm as described previously [23], the multi-ubiquitinated proteins in the TBC seem to be derived also from the nucleus of step 19 spermatids. M-Ub signals are associated with not only dense material surrounding the tubules but also the lumen of them. If multi-ubiquitinated proteins are derived from the spermatid nucleus as well as cytoplasm, they should be transported across two plasma membranes of spermatids and Sertoli cell. Although the transport mechanism is quite unclear, the present results suggest that proteins which become unnecessary with rapid and extensive reduction of the cytoplasm as well as compaction of the nucleus are degraded not only by lysosomal system but also by ubiquitin-proteasome system.

\section{Acknowledgements}

The work was supported by the university research fund, in part by a grant-in-aid (17570158) from the Ministry of Education, Science, Culture and Sport, and by the Science Research Promotion Fund from the Promotion and Mutual Aid Corporation for Private Schools of Japan.

\section{REFERENCES}

[1] S. S. Guraya, "Biology of Spermatogenesis and Spermatozoa in Mammals,” Springer-Verlag, Berlin, 1987. http://dx.doi.org/10.1007/978-3-642-71638-6

[2] D. M. de Krester and J. B. Kerr, "The Cytology of the Testis," In: E. Knobil and J. Neill, Eds., The Physiology of Reproduction, Raven Press, New York, 1988, pp. 837932.

[3] Y. Clermont and A. Rambourg, "Evolution of the Endoplasmic Reticulum during Rat Spermiogenesis," American Journal of Anatomy, Vol. 151, No. 2, 1978, pp. 191211. http://dx.doi.org/10.1002/aja.1001510204

[4] M. H. Burgos and D. W. Fawcett, "Studies in the Fine Structure of the Mammalian Testis. I. Differentiation of the Spermatids in the Cat (Felis Domestica)," Biophysical Biochemical Cytology, Vol. 1, No. 4, 1955, pp. 287-315. http://dx.doi.org/10.1083/jcb.1.4.287

[5] A. Abou-Halia and R. P. Tulslani, "Mammalian Sperm Acrosome: Formation, Contents, and Function," Archive Biochemistry and Biophysics, Vol. 379, No. 2, 2000, pp. 173-182. http://dx.doi.org/10.1006/abbi.2000.1880

[6] D. W. Fawcett and D. M. Phillips, "The Fine Structure and Development of the Neck Region of the Mammalian Spermatozoon," Anatomical Record, Vol. 165, No. 2, 1969, pp. 153-184. 
http://dx.doi.org/10.1002/ar.1091650204

[7] W. S. Ward and D. S. Coffet, "DNA Packaging and Organization of in Mammalian Spermatozoa: Comparison with Somatic Cells," Biology of Reproduction, Vol. 44, No. 4, 1991, pp. 569-574. http://dx.doi.org/10.1095/biolreprod44.4.569

[8] W. S. Ward, "Function of Sperm Chromatin Structural Elements in Fertilization and Development," Molecular Human Reproduction, Vol. 16, No. 1, 2010, pp. 30-36. http://dx.doi.org/10.1093/molehr/gap080

[9] R. M. Sharpe, S. M. Maguire, P. T. K. Saunders, M. R. Millar, L. D. Russell, D. Garten, S. Bachmann, L. Mullins and J. J. Mullins, "Infertility in a Transgenic Rat Due to Impairment of Cytoplasmic Elimination and Sperm Release from the Sertoli Cells," Biology of Reproduction, Vol. 53, No. 1, 1995, pp. 214-226. http://dx.doi.org/10.1095/biolreprod53.1.214

[10] A. K. Rengan, A. Agarwal, M. van der Linde and S. S. du Plessis, "An Investigation of Excess Residual Cytoplasm in Human Spermatozoa and Its Distinction from the Cytoplasmic Droplet," Reproductive Biology and Endocrinology, Vol. 10, 2012, pp. 2-8.

http://dx.doi.org/10.1186/1477-7827-10-92

[11] C. S. Sapsford and C. A. Rae, "Ultrastructural Studies on Sertoli Cells and Spermatids in the Bandicoot and Ram during the Movement of Mature Spermatids into Lumen of the Seminiferous Tubule," Australian Journal of Zoology, Vol. 17, No. 3, 1969, pp. 415-445. http://dx.doi.org/10.1071/ZO9690415

[12] J. P. Fouquet, "La Spermiation et la Formation des Corps Résiduels chez le Hamster: Role des Cellules de Sertoli," Journal de Microscopie, Vol. 19, No. 2, 1974, pp. 161168.

[13] D. W. Fawcet, "Ultrastructure and Function of the Sertoli Cell," In: D. W. Hamilton and R. O. Greep, Eds., Handbook of Physiology. Male Reproductive System, Vol. V, Section 7, Endocrinology, American Physiological Society, Washington DC, Waverly Press Inc., Baltimore, 1975, pp. 21-55.

[14] L. Russell and Y. Clermont, "Anchoring Device between Sertoli Cells and Late Spermatids in Rat Seminiferous Tubules," Anatomical Record, Vol. 185, No. 3, 1976, pp. 259-278. http://dx.doi.org/10.1002/ar.1091850302

[15] L. D. Russell, "Spermatid-Sertoli Tubulobulbar Complexes as Devices for Elimination of Cytoplasm from the Head Region of Late Spermatids of the Rat," Anatomical Record, Vol. 194, No. 2, 1979, pp. 233-246. http://dx.doi.org/10.1002/ar.1091940205

[16] L. D. Russell, "Further Observations on Tubulobulbar Complexes Formed by Late Spermatids and Sertoli Cells in the Rat Testis," Anatomical Record, Vol. 194, No. 2,
1979 , pp. $213-232$

http://dx.doi.org/10.1002/ar.1091940204

[17] G. E. Mortimore and A. R. Pösö, "Lysosomal Pathway in Hepatic Protein Degradation: Regulatory Role of Amino Acids," Federation Proceedings, Vol. 43, No. 5, 1984, pp. 1289-1294.

[18] W. A. Dunn Jr., "Autophagy and Related Mechanisms of Lysosome-Mediated Protein Degradation," Trends in Cell Biology, Vol. 4, No. 4, 1994, pp. 139-143. http://dx.doi.org/10.1016/0962-8924(94)90069-8

[19] A. Hershko and A. Ciechanover, "The Ubiquitin System for Protein Degradation," Annual Review of Biochemistry, Vol. 61, 1992, pp. 761-807. http://dx.doi.org/10.1146/annurev.bi.61.070192.003553

[20] M. Hochstrasser, "Ubiquitin, Proteasomes, and the Regulation of Intracellular Protein Degradation," Current Opinion in Cell Biology, Vol. 7, No. 2, 1995, pp. 315-223. http://dx.doi.org/10.1016/0955-0674(95)80031-X

[21] S. Yokota, "Preparation of Colloidal Gold Particles and Conjugation of Protein A, IgG, F(ab') $)_{2}$, and Streptavidin," In: S. D. Schwarzbach and T. Osafune, Eds., Immunoelectron Microscopy. Methods and Protocols, Springer, New York, 2012, pp. 109-119.

[22] L. D. Russell, R. A. Ettlin, A. P. S. Hikim and E. D. Clegg, "Histological and Histopathological Evaluation of the Testis," Cache River Press, Florida, 1990, pp. 59119.

[23] C. M. Haraguchi, T. Mabuchi, S. Hirata, T. Shoda, T. Tokumoto, K. Hoshi and S. Yokota, "Possible Function of Caudal Nuclear Pocket: Degradation of Nucleoproteins by Ubiquitin-Proteasome System in Rat Spermatids and Human Sperm," Journal of Histochemistry and Cytochemistry, Vol. 55, No. 6, 2007, pp. 585-595. http://dx.doi.org/10.1369/jhc.6A7136.2007

[24] R. L. Sparando and L. D. Russell, "Comparative Study of Cytoplasmic Elimination in Spermatids of Selected Mammalian Species," American Journal of Anatomy, Vol. 178, No. 1, 1987, pp. 72-80. http://dx.doi.org/10.1002/aja.1001780109

[25] D. W. Fawcett and D. M. Phillips, "Observation on the Release of Spermatozoa and Changes in the Head during Passage through the Epididymis," Journal of Reproduction and Fertility, Suppl. 6, 1969, pp. 405-418.

[26] J. B. Kerr and D. M. Kretser, "The Role of the Sertoli Cell in Phagocytosis of Residual Bodies of Spermatids," Journal of Reproduction and Fertility, Vol. 36, 1974, pp. 439-440. http://dx.doi.org/10.1530/jrf.0.0360439

[27] K. Tanaka, "The Proteasome: Overview of Structure and Functions," Proceedings of Japan Academy, Series B, Physical and Biological Sciences, Vol. 85, No. 1, 2009, pp. 12-36. http://dx.doi.org/10.2183/pjab.85.12 